Objective. To present a case of a 79-year-old male with frontal lobe dementia (following a cerebral abscess) who was referred due to inappropriate sexualised behaviour (ISB) in a care home setting.

To discuss the evidence base for the management of ISB in frontotemporal dementia.

Case report. 79-year-old male patient who was diagnosed with frontal lobe dementia, following a craniotomy to aspirate and evacuate a cerebral abscess which affected the left frontal, parietal and temporal lobes. He then started to exhibit sexualised behaviour; he was using sexualised language towards female residents and care workers in the residential home, and was inviting residents to his room and asking them to touch him. This behaviour was felt to be due to inappropriate sexual behaviour which forms part of the spectrum of behavioural and psychological symptoms of dementia. Non-pharmacological interventions were tried but failed to manage his symptoms. He was started on Paroxetine which treated the symptoms for approximately 12 months. The symptoms reocurred and he was switched to Amisulpride which had a positive effect on his symptoms.

Discussion. ISB is a behavioural and psychological symptom of dementia and may be seen in $7 \%$ to $25 \%$ of patients with dementia. ISB is distressing for the caregivers and also presents considerable challenges for the treating clinician. ISB presents with behaviour such as sexual language, implied sexual acts, and overt sexual acts. A differentiation should be made between whether the act was one of intimacy-seeking or disinhibition. However, there is a need to intervene when there are risks to the wellbeing and safeguards of the patient and also caregivers and residents. ISB can be difficult to treat, and there is limited evidence on the subject. It is often better managed by non-pharmacological interventions if possible, due to patients often being less responsive to psychoactive therapies and the risks involved with using medication. Non-pharmacological interventions include environmental, behavioural and educational approaches, and examples of these are discussed. Pharmacological interventions are also discussed, but there is a lack of evidence in this area; currently the evidence is from case series and case reports. The variety of drug classes illustrate the non specific nature of drug therapy.

Conclusion. Managing and treating ISB is difficult and complex.

The evidence suggests using non-pharmacological approaches as first line before considering pharmacological interventions.

However, there is a need for further research to develop robust non-pharmacological and pharmacological interventions in the treatment of ISB.

A rare case of a patient with resistant schizophrenia who hears a voice reading the texts instead of being read in her mind

\author{
Thilini Nanayakkara* and Vipula Wijesiri \\ Teaching Hospital Peradeniya \\ ${ }^{\star}$ Corresponding author.
}

doi: 10.1192/bjo.2021.344

Objective. This case is presented to describe a rare psychopathology in which the patient hears her own voice speaking out loud all the texts that she sees in books or papers and she cannot read them inside her mind. This psychopathological phenomena has some features of reflex hallucinations, thought echo as well as of inner reading voices yet it cannot be categorized into either one.

Case report. This is a 26-year-old female with Schizophrenia for 3 years. While on medication 8 months before presentation she started hearing her own voice reading any text that she sees. When she sees a text she cannot read it in her mind and understand, but she hears it in her own voice to her ears. With this she also hears other voices talking about her and to her. She also believes that her father is the one who controls all her actions and the things that happen to her. In her mental state examination her mood was euthymic and she had delusions of control, thought broadcasting and in her perceptions she had visual perceptual abnormality where she saw the same object she would look at in another direction but they are under her control. She also had second and third person auditory hallucinations. She was admitted to start on clozapine because her voices did not respond to any medication.

Discussion. Auditory hallucinations are the most commonly encountered type in schizophrenia with a prevalance of $70-80 \%$. This patient hears the words that she sees which has some features of reflex hallucinations, however in the latter the hallucination is not a transformation of the perception. This also has some qualities of thought echo, where just as the patient thinks she can hear them. However in this patient she cannot read the texts in her mind. Inner reading voices are where a person talks to oneself while reading, however in the subjective mind. In our patient this phenomenon also proved to be the most difficult to treat as all her other auditory hallucinations responded to Clozapine, while still this phenomenon remained.

Conclusion. This case is presented to describe the rare psychopathology in this patient in the form of auditory hallucinations

\section{Characterization of a novel CSF1R mutation causing hereditary diffuse leukoencephalopathy with spheroids in a case presenting with young-onset dementia}

Walid Nasr ${ }^{1 *}$, Mahmoud Gad ${ }^{1}$ and Tareq Qassem ${ }^{2}$

${ }^{1}$ Al-Amal Hospital, Ministry of Health and Prevention and

${ }^{2}$ Maudsley Health, Al-Amal Hospital, Ministry of Health and Prevention, Mohammed Bin Rashid University Of Medicine and Health Sciences, Institute of Psychiatry, Ain Shams University ${ }^{\star}$ Corresponding author.

doi: 10.1192/bjo.2021.345

Objective. This poster aims to report an unregistered mutation CSF1R gene in a patient presenting young-onset dementia.

Hypothesis: Novel heterozygous deletion-insertion mutation in the Colony-Stimulating Factor 1 Receptor (CSF1R) gene is linked to a case of hereditary diffuse leukoencephalopathy with spheroids (HDLS), presenting with young-onset dementia.

Background. CSF1R mediates proliferation, differentiation, and survival of monocytes/ macrophages and microglia. Pathogenic variants in the CSF1R gene cause autosomal dominant diffuse hereditary leukoencephalopathy with spheroids characterized by variable behavioural, cognitive, and motor changes, usually presenting with young-onset dementia. The average lifespan after the start of the symptoms is often 6 years.

Case report. Molecular genetic analysis of whole-exome sequencing (WES) was carried out for a 49-year-old male patient presenting with rapid cognitive decline, behavioural symptoms and impaired sphincteric control.

Discussion. WES identified the heterozygous deletion-insertion variant c.2356_2357delinsAC p.(Leu786Thr) (chr5:14943586749435868; hg19) in the CSF1R gene. To the best of our knowledge the variant has not been described in the literature so far (HGMD 2019.3). No allele frequencies in the general population have been documented.

Conclusion. We believe that we have identified a novel mutation in the CSF1R gene. This mutation is likely to be linked to this patient presenting with young-onset dementia. 
Treatment with the direct oral anticoagulants (DOACs) Apixaban and Rivaroxaban associated with significant worsening of behavioural and psychological symptoms of dementia (BPSD)

Kim Porter $^{1 \star}$, Stephen De Souza ${ }^{1}$, Iain Hargreaves ${ }^{2}$ and Rebecca Goddard ${ }^{3}$

${ }^{1}$ Somerset NHS Foundation Trust; ${ }^{2}$ University College London and

${ }^{3}$ Avon and Wiltshire Partnership Trust

${ }^{\star}$ Corresponding author.

doi: 10.1192/bjo.2021.346

Objective. To demonstrate the increasing evidence for an aetiological role of cerebral mitochondrial dysfunction in neuropsychiatric disorders.

To raise awareness of the importance of frontline staff partaking in post marketing surveillance of medications.

Case report. We report the cases of two patients who developed worsening BPSD, coinciding with starting the factor Xa inhibitor DOAC medications Apixaban and Rivaroxaban respectively. Both patients required detaining under the Mental Health Act (MHA). Their symptoms improved significantly, within two weeks, on switching to alternative anticoagulant therapies and they were both discharged from the acute psychiatric ward.

Discussion. Frontline healthcare staff in acute settings and the community manage a heavy workload. It is all too easy to overlook potential neuropsychiatric drug side effects, especially if they are not clearly listed. They may be easily missed amongst older patients and wrongly attributed to dementia.

Rivaroxaban is structurally related to the antibiotic Linezolid which has been reported to cause mitochondrial toxicity. Pre marketing In vitro studies concluded the risk of mitochondrial toxicity associated with this anticoagulant to be low. However a more recent in vitro study, using rat kidney mitochondria, reported evidence of mitochondrial swelling and a collapse of the membrane potential following exposure to low doses of Rivaroxaban. The effect of Apixaban, which is structurally related to Rivaroxaban, has yet to be investigated on mitochondrial function.

Recent research supports not only an association between reduced cerebral mitochondrial function and neuropsychiatric symptoms and disorders, but also the aetiological role it may play.

There is a need for a far greater awareness and understanding of the potential cerebral mitochondrial toxicity of drugs commonly prescribed to our older populations.

Conclusion. Cerebral mitochondrial toxicity can have a significant impact on the health and well-being of patients.

Older patients are particularly prone to experiencing neuropsychiatric side effects that may not have been apparent during preclinical trials.

Development of a rating scale of drugs that are potentially less toxic to cerebral mitochondria could inform national prescribing guidelines and enable safer treatments to be offered to older people, reducing the likelihood of them experiencing apparent behavioural and psychological symptoms of dementia.

Dyeing to live - a case of clozapine in disguise, and physicians' courage

Alma Rae ${ }^{1 \star}$ and Thomas Kinghorn ${ }^{2}$

${ }^{1}$ Locum Consultant Psychiatrist and ${ }^{2}$ NHS Lothian

${ }^{\star}$ Corresponding author.

doi: 10.1192/bjo.2021.347
Objective. ... in which clozapine tablets were dyed pink, to work around a delusion preventing treatment, and physicians tolerated and monitored an alarming early response to the drug.

Patient. 56-year-old female with severe enduring Bipolar I Disorder, current episode manic with psychosis, already an inpatient for six months. When first seen by us, polypharmacy was evident including haloperidol $25 \mathrm{mg}$ daily. Thorough trials of mood stablisers and second generation antipsychotics in various combinations had all failed. She had never had a clozapine trial.

MSE. Dishevelled middle-aged woman of European descent. Restless; shuffling gait; speech pressured, rapid and whispering, often to the point of unintelligibility. Affect labile: anxious and distressed, suspicious, angry, elevated and demanding. Thought form tangential+++ content paranoid persecutory themes, preoccupied with sexual trauma and delusional belief that yellow medication whether solid or liquid was poisonous. Risks of vulnerability, falls, aggression, neuroleptic malignant syndrome (NMS) and protracted psychotic mania requiring long term hospitalisation.

Plan. Change to clozapine.

Problem. All formulations are yellow.

Solution. Team discussion, ethical analysis, clozapine tablets dyed with red vegetable dye.

Ethical analysis. Potential benefit to patient great; current medications not effective and NMS possibly developing; she was fully informed about clozapine with no attempt made to hide the identity of the now crimson tablets.

Outcome. Patient accepted the clozapine. Temperature, $\mathrm{C}$ reactive protein (CRP) and troponin were all normal at baseline but all rose above normal in week 1 of initiation. They peaked in week 3 and by week 4 were dropping, normalising completely within a few weeks. She was transferred to a medical ward for monitoring during weeks 2 and 3 of titration. There were no electrocardiogram changes, no chest pain, no signs of bowel obstruction and no evidence of agranulocytosis. Clinically, she remained well throughout except for the rise in temperature. Once the yellow medicine delusion receded she accepted undyed yellow tablets; the result was discharge home with her best mental state and level of functioning in 15 years.

Significance of this case. There are no cases in the literature that we could find where tablets had been dyed, or where clozapine had been persisted with when such rises in temperature, CRP and troponin occurred. This case illustrates both. The risks in our view were outweighed by the simple fact that clozapine was her only hope of a life worth living.

Lockdown and visual hallucinations in older people: a community perspective

Rim Roufael*

Health Education and Improvement Wales (HEIW)

${ }^{*}$ Corresponding author.

doi: 10.1192/bjo.2021.348

Objective. After COVID-19 was declared as a pandemic, different countries have enforced lockdowns, and shielding to mitigate the spread of the virus as preventing loss of lives was the priority.

Our aim is to look for possible explanations for increased rates of visual hallucinations presented to Community Mental Health Teams for Older People during the period of lockdown. Case report. A review of clinical cases presenting with new onset visual hallucinations to the Community Mental Health Teams for 